\title{
La place pour le concept d'État gardien en droit de I'environnement dans l'encadrement du secteur minier à Madagascar
}

Lynda Hubert Ta

\section{ABSTRACT}

In environmental law, the concept of custodian state generally assumes that the state is the guarantor of the interests of present and future generations, and of the implementation of the objective of intra- and intergenerational equity for sustainable development. The state is expected to protect people's rights to a healthy environment and to safeguard the natural heritage in order to transmit it as a legacy to future generations. In the context of the mining sector in Madagascar, researchers have shown that the state's role tends to be redefined so as to limit itself to facilitating mining investments, to the detriment of rights and public interest protection. In Madagascar, successive policies and strategies have displaced the state from regulation and management of its own natural resources. According to the principles of neo-liberal economy, its disengagement from the regulation of mineral resource exploitation would allow the development of favorable conditions to improve resource management and to reduce environmental impacts. However, this leaves little room for maneuver to ensure the protection of social and environmental rights that may be affected by the development of this sector. The article reflects on this contradictory situation, its origins and its stakes in Malagasy environmental law.

\section{RÉSUMÉ}

En droit de l'environnement, le concept de l'État gardien postule d'une manière générale que l'État est garant des intérêts des générations actuelles et futures, donc de la mise en œuvre de I'objectif d'équité intra et intergénérationnelle du développement durable. L'État est supposé protéger les droits des populations à un environnement sain et sauvegarder le patrimoine naturel en vue de le transmettre en héritage aux générations à venir. Or, dans I'encadrement du secteur minier à Madagascar, des chercheurs ont démontré que le rôle de l'État tend à être redéfini de façon à se limiter à faciliter l'investissement minier, au détriment de la protection des droits et de l'intérêt public. À Madagascar, les politiques et stratégies successives ont écarté l'État de la réglementation et la gestion de ses propres ressources naturelles. Suivant les principes néolibéraux, son désengagement de l'encadrement

\author{
Correspondence: \\ Lynda Hubert Ta \\ Université Laval, Faculté de droit \\ Québec, Canada \\ Email: Iynda.hubert-ta.1@ulaval.ca
}

de l'exploitation des ressources minières permettrait le développement de conditions favorables à I'amélioration de la gestion des ressources et à la diminution des impacts environnementaux. Cela lui laisse toutefois peu de marge de manœuvre pour promouvoir la protection des droits sociaux et environnementaux pouvant être affectés par le développement du secteur minier. L'article propose un début de réflexion sur cette situation contradictoire, ses origines et ses enjeux en droit de I'environnement malgache.

\section{INTRODUCTION}

Ce texte revêt une dimension prospective ; il s'agit d'un questionnement qui met à jour certaines idées demandant encore à être creusées. C'est à cette réflexion prospective que le lecteur est invité à travers cet essai dont l'objectif est de soulever des questions sur le rôle de l'État malgache dans la protection de I'environnement et sa capacité à assumer ce rôle dans l'encadrement du secteur minier. Il convient de noter que la conception de la protection de l'environnement que nous adoptons dans ce texte englobe une dimension sociale indissociable, que I'on retrouve d'ailleurs dans la plupart des textes en droit de l'environnement. Les deux aspects (environnemental et social) étant étroitement imbriqués, Iorsque nous évoquons la protection de I'environnement, cela implique aussi notamment la protection des communautés locales.

La réflexion s'appuie sur les processus sélectifs de libéralisation mis en place à Madagascar sous l'impulsion de divers acteurs, particulièrement les réformes successives dans le secteur de l'environnement et des ressources naturelles, notamment les ressources minières. En effet, la politique minière malgache de 1998 a été lancée dans le contexte de la mise en œuvre du programme de désengagement partiel de l'État de certains secteurs économiques entamé dans les années 1980, et du Projet de réforme du secteur minier (PRSM) promu et appuyé par la Banque Mondiale de 1998 à 2002 (Banque Mondiale 2003). Le PRSM mettait en place un ensemble de réformes du cadre légal et réglementaire du secteur minier, avec pour objectif la redéfinition et la limitation du rôle de l'État dans ce secteur, pour mieux offrir un 


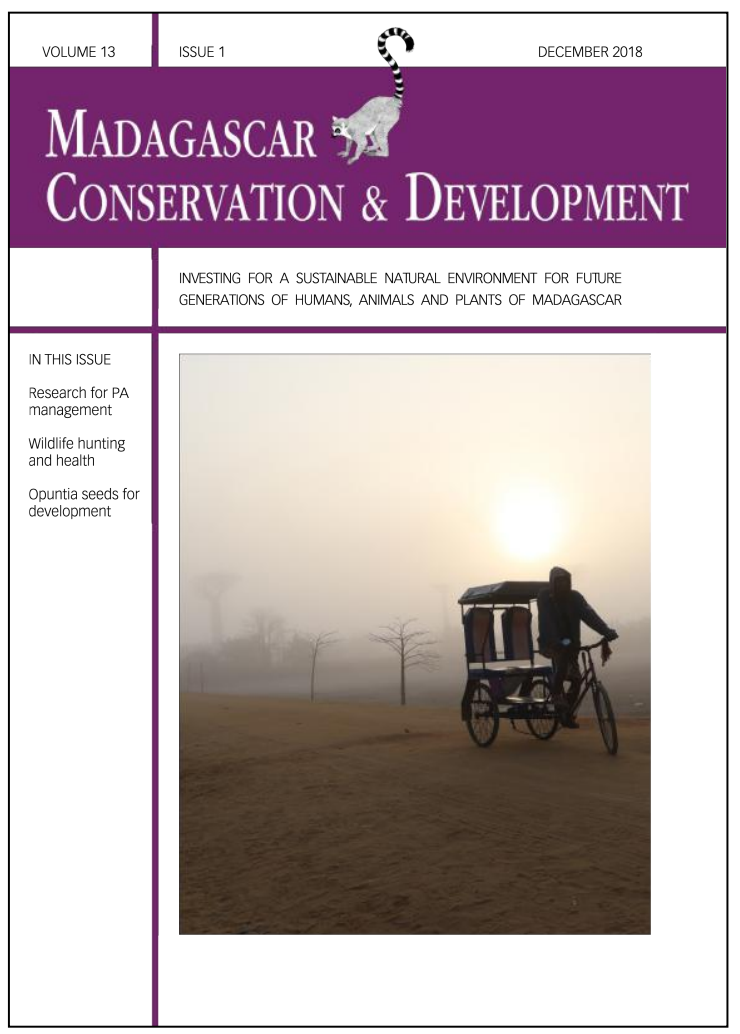

Madagascar Conservation \& Development is the journal of Indian Ocean e-Ink. It is produced under the responsibility of this institution. The views expressed in contributions to MCD are solely those of the authors and not those of the journal editors or the publisher.

All the Issues and articles are freely available at http://www.journalmcd.com

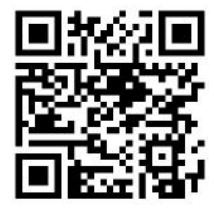

Contact Journal MCD

info@journalmcd.net for general inquiries regarding MCD funding@journalmcd.net to support the journal

Madagascar Conservation \& Development Institute and Museum of Anthropology

University of Zurich

Winterthurerstrasse 190

$\mathrm{CH}-8057$ Zurich

Switzerland

Indian Ocean e-Ink

Promoting African Publishing and Education

www.ioeink.com

Missouri Botanical Garden (MBG)

Madagascar Research and Conservation Program

Missouri Botanical Garden

BP 3391

Antananarivo, 101, Madagascar 
environnement légal favorable et incitatif aux investisseurs (Sarrasin 2006).

Les recherches menées par le Groupe de recherche sur les activités minières en Afrique (GRAMA) et le centre interdisciplinaire de recherche en développement international et société (CIRDIS) de I'Université du Québec à Montréal ont souligné l'impact de telles réformes en termes de réduction de l'espace de décision de l'État, de fragilisation de ses capacités de suivi et de contrôle, et son aptitude à apporter des mesures correctives (Campbell 2010). Par ailleurs, le transfert d'une part des fonctions et responsabilités de l'État en matière sociale et environnementale vers des acteurs privés n'est pas sans conséquence sur l'intérêt public, la protection des droits sociaux et environnementaux et les objectifs d'équité intra et intergénérationnelle du développement durable. Une telle situation suscite particulièrement des questionnements importants dans un pays tel que Madagascar, qualifié de hotspot de la biodiversité mondiale, concernant le rôle et les responsabilités publiques dans la protection du patrimoine naturel, dans l'intérêt des générations actuelles et futures. Notre démarche prospective nous a conduits à nous intéresser aux contributions du concept juridique d'« État gardien » en droit de l'environnement, pour alimenter ces réflexions. Nous avons donc tenté un premier survol de la littérature sur le concept d'État gardien afin de comprendre dans un premier temps les origines, les objectifs et la portée de ce concept. Puis, à travers l'examen de quelques textes de lois nous avons cherché à identifier les éléments de base du concept dans le droit de l'environnement malgache et ses implications possibles pour le secteur minier, notamment en termes d'obligations fiduciaires attribuées à l'État dans la protection de la biodiversité.

\section{LE CONCEPT D'ÉTAT GARDIEN EN DROIT DE L'ENVI- RONNEMENT}

Le concept d'État gardien n'est pas nouveau ; ses origines sont anciennes, même si le vocabulaire utilisé pour le décrire a varié selon les époques et les systèmes juridiques. De nos jours par exemple, on y rattache d'autres qualificatifs comme fiduciaire, mandataire, garant, public trust, stewardship ou patriae.

Concept hybride, ses origines sont situées à l'intersection entre le droit romain de l'antiquité et le droit anglo-saxon contemporain. II puise en effet dans les notions de patrimonium et de res communis, res publicae, res univertatis du droit romain antique, qui décrivent à différents degrés des choses (biens ou ressources) non-appropriables ou dont la propriété est reconnue à l'ensemble de la communauté, et dans la théorie du public trust en droit de common law, qui évoque un patrimoine (le trust ou la fiducie) qui est géré par une personne (le trustee ou fiduciaire) pour le bénéfice d'une autre personne (le bénéficiaire) (Jones 1992).

La doctrine du public trust ou fiducie publique appliquée au droit de l'environnement et des ressources naturelles a d'abord été développée par le juriste américain Joseph Sax en 1970 et a depuis fait l'objet de débats fournis dans la littérature juridique. D’une manière générale, Sax (1970) identifie trois éléments constitutifs d'une fiducie publique : (i) l'existence d'un bien public commun, (ii) la responsabilité d'assurer la protection de ce bien pour les générations actuelles et futures et (iii) la gestion publique dont le caractère désintéressé, transcendant des intérêts individuels, est à privilégier. À partir de là, on peut déduire la relation qui est à la base du concept d'État gardien ou fiduciaire : il réfère à un régime de protection et de gestion publique dans l'intérêt public et dont la finalité est l'équité intra et intergénérationnelle (BrownWeiss 1984, Baslar 1998). Il attribue à l'État la mission et la responsabilité de préserver le patrimoine naturel, afin qu'il puisse être transmis en héritage aux générations futures (Moench 2001). Les bénéficiaires, qui sont les générations actuelles et futures, ont le droit d'attendre de l'État qu'il prenne toutes les mesures nécessaires pour protéger le patrimoine et pour en assurer la gestion prudente et diligente (Wood 2014).

Une fois le concept défini, on peut aussi dégager certains principes qui fondent le concept et les effets qui s'y rattachent. II est intéressant de noter que les prérogatives octroyées par le concept se déclinent d'abord en devoirs en regard des objectifs d'intérêt public : le devoir de l'État de " prendre toute mesure nécessaire à la conservation du bien » (Vincent 2008), le devoir d'impartialité et de loyauté envers les bénéficiaires, le devoir de diligence, de précaution et de prévention, le devoir d'intégration des considérations environnementales à l'ensemble des politiques publiques, le devoir de préserver le droit à un environnement sain et les droits fondamentaux des bénéficiaires de la ressource, la responsabilité pour inaction en cas de dommages environnementaux, le devoir de prévoir des mécanismes de plaintes, de reddition de comptes, de transparence, etc. sont autant d'obligations qui sont soulignées dans la littérature (Fort 2012). Ces obligations permettent de souligner l'importance de renforcer les compétences réglementaires de l'État dans la définition, la planification et la mise en œuvre des règles devant permettre de parvenir aux objectifs de protection. II est également intéressant de comprendre les implications de ces responsabilités étatiques, comme le droit des bénéficiaires à demander réparation au fiduciaire pour les détériorations du bien, le droit à l'information, à la participation.

Le concept d'un État gardien de l'environnement et des ressources naturelles présenterait, semble-t-il, l'intérêt de renouveler la réflexion sur le rôle de l'État, la légitimité de ses interventions au regard de sa mission d'intérêt public et de sa souveraineté sur ses ressources naturelles, ainsi que sur sa responsabilité dans la mise en œuvre de l'objectif d'équité du développement durable. II se distingue de la conception traditionnelle de l'État centralisateur dans la mesure où il propose de baliser le pouvoir discrétionnaire de l'État par une gestion consensuelle et la mise en place de mécanismes d'imputabilité, permettant la surveillance et le contrôle par le public de l'usage des ressources. Par ailleurs, la répartition des pouvoirs de l'État vers des instances publiques plus restreintes, plus territoriales (États fédérés, provinces, municipalités) est une autre idée proposée dans la littérature pour encadrer l'autorité publique (Girard 2012).

L'idée véhiculée par le concept, selon laquelle la nature constitue un patrimoine commun à préserver au nom d'intérêts collectifs, est aussi présente dans certains droits nationaux, régionaux et en droit international. Par exemple, à l'échelle internationale, la Convention pour la protection du patrimoine mondial, culturel et naturel de I'UNESCO et la Convention des Nations Unies sur le droit de la mer réaffirment le principe d'un « patrimoine commun de I'humanité » à l'égard de certaines ressources sensibles, écosystèmes fragiles ou d'une valeur inestimable pour les générations actuelles et futures. À l'échelle régionale, le droit communautaire européen considère que les oiseaux migrateurs constituent un "patrimoine commun " sous la garde de I'Union Européenne et qu'il en résulte une obligation de résultat en matière de protection de la ressource (Directive concernant la 
conservation des oiseaux sauvages, préambule, $3^{e}$ et $8^{e}$ considérants). Enfin, à l'échelle provinciale, la Loi québécoise sur l'eau par exemple, affirme que les ressources en eau sont le " patrimoine commun de la nation québécoise ", qu'elles revêtent un " caractère collectif », et que l'État québécois en est le gardien et doit ainsi s'assurer que les utilisations de la ressource par les personnes privées n'affectent pas la ressource et les usages collectifs.

Compte tenu de l'ampleur des atteintes que peuvent porter les activités du secteur minier au patrimoine naturel de pays riches en biodiversité comme Madagascar, il est pertinent de questionner l'ancrage ou l'application du concept d'État-gardien dans l'encadrement de ce secteur spécifique.

\section{L'HYPOTHĖSE D'UN ÉTAT GARDIEN ET LE SECTEUR MINIER À MADAGASCAR : SUR QUELLE BASE ET QUELS ENJEUX?}

UNE NOTION DE PATRIMOINE COMMUN À GARDER DANS LE DROIT DE L'ENVIRONNEMENT MALGACHE. La biodiversité malgache est I'une des plus riches au monde, avec des écosystèmes, une flore et une faune à très grande endémicité : $85 \%$ de la flore, $39 \%$ des oiseaux, $91 \%$ des reptiles, $99 \%$ des amphibiens et $100 \%$ des lémuriens sont endémiques à la Grande île (Goodman et Benstead 2005). Les écosystèmes forestiers sont les plus riches mais aussi les plus menacés par les activités humaines et certains d'affirmer que la perte d'un hectare de forêt à Madagascar a un effet irréversible sur la biodiversité mondiale (Hufty et Muttenzer 2002). Une partie de cette richesse naturelle est aujourd'hui considérée comme faisant partie du patrimoine mondial de I'UNESCO, dont deux sites naturels d'une grande importance figurant sur la Liste du patrimoine mondial et huit autres sites figurant sur la Liste indicative (UNESCO 2017). À l'échelle internationale, Madagascar est partie à un certain nombre d'instruments internationaux dédiés à la protection de la biodiversité et qui la consacrent comme un patrimoine naturel commun à préserver. C'est le cas, par exemple, du Protocole relatif aux zones protégées ainsi qu'à la faune et à la flore sauvages dans la région de l'Afrique orientale. La création et le maintien d'aires protégées constituent un des moyens privilégiés pour promouvoir la protection de la biodiversité. Lors du cinquième Congrès mondial des parcs de I'UICN en 2003, Madagascar s'est engagé à tripler la surface de ses aires protégées avec pour objectif d'atteindre six millions d'hectares en 2012, soit environ $10 \%$ du territoire national, un objectif qui a depuis été atteint (Gouvernement de Madagascar 2016).

Dans le droit interne, l'idée de la biodiversité malgache comme un patrimoine naturel à préserver pour les générations actuelles et futures (par exemple, pour les communautés locales dépendantes de ces ressources, dont les droits d'usage sont conservés, ou encore pour les membres de la communauté scientifique nationale et internationale) se retrouve aussi dans plusieurs textes stratégiques et juridiques, dont la plus importante est sans doute la Charte de l'environnement malgache qui parle d'un « patrimoine Malagasy de biodiversité », déclare que «[l]'environnement constitue une préoccupation prioritaire de l'État » et que « [l]a protection et le respect de l'environnement sont d'intérêt général » (Charte de l'environnement malgache, art 6, 3 et 4). On peut lire dans la Politique nationale de l'environnement (PNE) que ce "patrimoine revêt un intérêt particulier pour la communauté scientifique », qu'« en raison de son caractère unique dans le monde, le patrimoine environnemental malgache est considéré comme patrimoine de l'Humanité » dont la sauvegarde doit être faite « pour le bien être de la population malgache et celui des générations futures » (Charte de l'environnement malgache, titre II, chapitre 1, section 2).

UN PATRIMOINE NATUREL AFFECTÉ PAR LE DÉVELOPPEMENT MINIER. Or, ce patrimoine national et mondial est aujourd'hui plus que jamais touché par un secteur minier en pleine expansion; ces deux espaces (exploitation minière et conservation) venant à se superposer. Plus particulièrement, les aires de conservation sont directement affectées, comme c'est le cas de la forêt de Mikea dans le Sud-Ouest de l'île, d'une très grande importance socioéconomique et culturelle pour les communautés locales, laquelle pourrait-être en partie déboisée par la construction de routes destinées à acheminer l'ilménite extrait par le projet Toliara Sands vers le port de Toliara (Rakotondrainibe 2015). À I'Ouest, la construction d'infrastructures routières et portuaires nécessaires au projet d'extraction de fer de la compagnie chinoise Madagascar Wuhan Iron and Steel Corporation ont fait également naître des inquiétudes pour le Parc national de la baie de Baly à Soalala (Valis 2012). Parmi d'autres exemples, I'exploration de terres rares par Tantalus Rare Earths sur la presqu'île d'Ampasindava s'étendrait sur $20 \mathrm{~km}^{2}$ de forêts intactes, à proximité de la nouvelle aire protégée d'Ampasindava-Galoko-Kalobinono, elle-même entourée par environ six autres compagnies minières (Gouvernement de Madagascar 2015). Ensuite, plusieurs milliers d'hectares de forêts dont des forêts humides intactes de la région Alaotra-Mangoro abritant une multitude d'espèces endémiques ou parmi les plus menacées de lî̂le, sont aussi affectés par la mine de nickel et le pipeline du projet Ambatovy (Projet Ambatovy 2006). Enfin, le projet d'exploration de sables bitumineux à Tsimiroro et Bemolanga par Madagascar Oil pourrait présenter des risques pour la biodiversité, avec trois aires protégées à proximité : Ambohijanahary, le Tsingy de Beanka et le fameux Tsingy de Bemaraha, inscrit au Patrimoine mondial de I'UNESCO depuis 1990 (Les Amis de la Terre France 2012, Waeber et al. 2015).

QU'EN EST-IL DU DEVOIR DE PROTECTION ET DU RÔLE DE L'ÉTAT MALGACHE ? La multiplication de telles situations soulève un certain nombre de questions sur la façon dont ce conflit d'intérêt est abordé dans la réglementation des impacts du secteur minier et fait surgir des enjeux concernant les discours avancés et la capacité de l'État à percevoir d'abord puis à investir le rôle de gardien. En effet, si le droit positif malgache affirme haut et fort qu'il y a un patrimoine environnemental à protéger, un objectif qui est maintes fois rappelé par les instruments légaux et réglementaires encadrant le secteur minier comme les codes miniers de 1999 et de 2005 (titre 5, chapitre 2) et la Charte de l'environnement malagasy actualisée de 2015 (préambule et article 3, 1er objectif), le droit n'est pas aussi clair sur le rôle que l'État doit jouer et les moyens à sa disposition pour mettre en œuvre cet objectif. Plusieurs autres dispositions législatives alimentent cette confusion. Par exemple, dans un premier temps, la Loi constitutionnelle $n^{\circ}$ 2007-001 du 27 avril 2007 déclare dans son article 37 que «l'État garantit la liberté d'entreprise dans la limite du respect de l'intérêt général, de l'ordre public, des bonnes mœurs et de l'environnement ", ce qui semble accorder à l'État un certain rôle de garde-fou en faveur de l'environnement. Mais, dans un deux- 
ième temps, selon l'article 7 de la Charte de l'environnement de 1990, la protection de l'environnement concerne un large éventail d'acteurs comme l'État, bien entendu, mais aussi les opérateurs économiques. De plus, la Politique nationale de l'environnement insiste sur la nécessité pour l'État de se désengager et de « céder sa place aux opérateurs privés » comme "des associations d'usagers, les ONG et des entreprises privées appelées à préserver et mettre en valeur les ressources du pays "; son rôle doit se limiter à " développer les incitations nécessaires, (...) suivre et évaluer les actions sur le terrain » (Charte de l'environnement malgache de 1990, titre II, chapitre III, ii). Dans cette optique, le Plan d'action environnementale a intégré explicitement les principes de l'ajustement structurel, de la décentralisation et de libéralisation dans la mise en œuvre de la Politique nationale de l'environnement (Charte de l'environnement malgache de 1990, titre III, chapitre 1, §§ 3.2, 3.3 et 3.5).

Quelques-unes des implications de ce régime en termes d'impacts sociaux et environnementaux des projets miniers peuvent être alors soulignées. Tout d'abord, une des stratégies choisies pour tenter de concilier ces extrêmes semble résider dans un glissement de la prise en charge de la protection de l'environnement qui s'est opéré de la sphère publique vers la sphère privée, cette dernière étant présentée comme la mieux placée pour prendre en charge les coûts des dégradations environnementales que le discours dominant associe à la pression démographie et aux pratiques agricoles des communautés locales (Sarrasin 2005). Ce transfert de compétences est consacré, par exemple, par la Loi n 2001/05 portant Code de gestion des aires protégées qui convient dans ses articles 4 et 31 que la gestion des aires protégées peut être confiée à un organisme autonome, public ou privé. C'est ainsi que parmi les mesures d'atténuation de leurs impacts environnementaux, des projets miniers comme le Projet Ambatovy ou le projet de QIT Madagascar Minerals ont mis en place des programmes pour la conservation de la biodiversité malgache, à travers la création d'aires protégées dont le financement et la gestion sont confiés aux compagnies minières. Ce qui n'est pas sans conséquence sur les communautés locales puisqu'on peut s'attendre à ce que la gestion par les acteurs privés soit, à priori, différente de celle de l'État dont la mission d'intérêt public suppose la prise en compte et la protection des droits d'usage traditionnels sur la ressource, cela même si dans la pratique les deux types de gestion peuvent comporter des lacunes. Dans le cas de l'aire protégée sous la gestion du projet Ambatovy, le Collectif pour la défense des terres malgaches rapporte que des cultivateurs de cresson provenant des communautés avoisinantes avaient besoin d'accéder à la rivière comprise dans les limites de l'aire protégée pour leurs activités, qu'ils exerçaient depuis des dizaines d'années. Certains d'entre eux auraient été arrêtés pour avoir introduit une espèce végétale exogène (le cresson) dans l'aire protégée, puis relâchés sous réserve de cesser leurs activités (Rakotondrainibe 2015). Pour ce qui est de l'aire protégée gérée par la compagnie QIT Madagascar Minerals dans le Sud-Est de l'île, la situation peut sembler paradoxale si I'on considère que parmi les zones visées par le projet d'extraction d'ilménite, les sites de Petriky et Sainte Luce ont vu la création d'aires protégées confiées à la gestion de l'entreprise. Dans ce cas, cette gestion a été marquée par la restriction de I'accès à ces zones par les communautés locales (Randriamiarantsoa 2015). La question de l'accès à la terre et aux ressources naturelles qui semble relier les enjeux de la conservation et du développement minier à Madagascar a été soulevée et est toujours d'actualité (Seagle 2013).

Par ailleurs, un rapport des Amis de la Terre après une enquête sur le terrain en 2012 souligne la faiblesse des capacités scientifiques, matérielles, financières et humaines des institutions publiques dans l'appréciation et l'évaluation des impacts environnementaux, ainsi que dans le contrôle et le suivi des pratiques des compagnies, qui ne permet pas d'assurer la préservation du patrimoine naturel commun. Comme l'explique le rapport : " [e]n dépit d'un cadre législatif et réglementaire apparemment contraignant en matière environnementale, les pouvoirs publics ont de grandes difficultés à faire respecter les obligations qu'ils ont eux-mêmes édictées » (Les Amis de la Terre France 2012). Ainsi, si la loi malgache exige que le promoteur obtienne un permis environnemental avant le permis d'exploration ou d'exploitation, plusieurs permis d'exploitation auraient été délivrés durant la crise de 2009 et au cours de la période de transition qui a suivi jusqu'en 2014, pendant laquelle les capacités de l'État malgache étaient les plus affaiblies (Les Amis de la Terre France 2012). Cette période a vu la suspension de l'aide internationale et la mise en place d'un gouvernement de transition dont la légitimité, les fonctions et les responsabilités mêmes n'étaient pas clairement établies. Dans ce contexte d'affaiblissement des capacités institutionnelles, les générations futures mais surtout actuelles, bénéficiaires présumés de la préservation du patrimoine, deviennent les grands perdants : selon le rapport des Amis de la Terre, privées de leur droits traditionnels sur les ressources en plus des atteintes à leur droit à un environnement sain, mal informées, " osant rarement se plaindre par peur des représailles, et ne bénéficiant pas de la protection des autorités, les populations se retrouvent donc face à face avec les multinationales, qui cherchent régulièrement à faire taire, ou à contrôler, les oppositions potentielles émanant de la société civile » (Les Amis de la Terre France 2012).

En bref, il semble que le droit soit nuancé sur le rôle de gardien que devrait jouer l'État malgache dans la préservation de son patrimoine naturel. Les textes juridiques font ressortir un rôle partagé et ne lui reconnaissent qu'un rôle limité de promotion et de coordination, ce qui n'est pas sans équivoque sur le partage des responsabilités et l'imputabilité des différents acteurs impliqués, au regard des bénéficiaires de ce patrimoine. Toutefois, ce contexte renforce l'idée d'explorer l'application de la théorie de la fiducie publique. En effet, selon Catherine Ribot : « la théorie de la fiducie publique ne soutiendrait l'avancée du droit protecteur des ressources naturelles que lorsque l'État est privé des instruments traditionnellement reconnus pour justifier ses interventions en matière d'intérêt général » (Ribot 2012), ce qui semble bien être le cas à Madagascar, suite aux réformes successives de libéralisation dans le secteur des ressources naturelles. L'intégration du concept dans le droit malgache pourrait alors contribuer à renforcer les mécanismes de protection des ressources naturelles.

\section{LIMITES AU CONCEPT ET À L'IDÉE D'UNE APPLICA- TION À MADAGASCAR}

Une première limite, parmi les plus soulevées par la littérature, concerne la confusion des critères et méthodes de qualification du patrimoine commun et son État gardien, une confusion qui peut se manifester à travers la grande variété des termes utilisés, les textes de droit parlant par exemple de « patrimoine commun 
de I'humanité ", " patrimoine commun des êtres humains ", de " patrimoine commun de la nation » (Paquerot 2012). Il en résulte un régime juridique tout aussi confus, mal défini, entre appropriation et non appropriation, consensualisme et réglementation. L'effectivité du concept pose également problème : il est intégré dans le droit positif mais souvent sous la forme de déclarations qu'il n'est pas aisé de transcrire en règles strictes et dont les effets juridiques demeurent relatifs (Paquerot 2012). Par ailleurs, comme le souligne Jacqueline Morand-Deviller (2012), I'ordre économique mondial pèse de tout son poids dans la balance et il arrive souvent que des ressources déclarées " patrimoine commun » car non rentables ou encore inexploitables changent de statut dès que les progrès de la science les rendent exploitables. L'autre faiblesse la plus fréquemment soulignée dans la littérature se rapporte à la détermination de la personnalité juridique des bénéficiaires du patrimoine (l'humanité, la nation, le peuple ou les générations futures), qui pose la question du droit d'action devant les tribunaux en cas de dommages environnementaux (Fort 2012).

Si le concept comporte plusieurs faiblesses, il a tout de même le mérite de susciter un renouvellement du débat sur la question de la conservation environnementale et du rôle que I'État peut ou doit jouer. II permet aussi de questionner les rapports juridiques d'appropriation des ressources naturelles, d'intégrer les enjeux liés au caractère limité de ces ressources et d'appréhender le partage des responsabilités dans la gestion durable des ressources (Fort 2012). Ainsi, lorsqu'envisagé dans le contexte de Madagascar, il permet de raviver le débat sur les questions de capacités (indissociables de celles des responsabilités), surtout politiques, institutionnelles et normatives, et de légitimité de l'action publique dans la gouvernance du secteur minier. Il offre par ailleurs une avenue de réflexion concernant un changement de paradigme dans l'encadrement du développement minier dans le pays. C'est aussi un concept qui a l'avantage d'être évolutif, flexible et dynamique, qui peut donc être adapté aux différents contextes, aux besoins et circonstances changeants de la protection du patrimoine naturel (Slade 2009). À ce propos, Jacqueline Morand-Deviller souligne que le concept se veut mobilisateur face à la généralisation de la « logique productiviste ", qu'il " permet de mettre en évidence les valeurs éthiques propres à l'ordre écologique qui ne sont pas les mêmes que celles de l'ordre économique » et " peut inspirer le choix des institutions et des méthodes propres à répondre à la fonction particulière des biens ». En quelque sorte, il s'agirait selon elle de mettre de l'avant " une nouvelle manière de gérer les biens du patrimoine commun et de rappeler aux gardiens de ces biens les devoirs qui leur incombent » (Morand-Deviller 2012).

\section{CONCLUSION}

S'agissant du patrimoine commun naturel, certains juristes n'hésitent pas à suggérer un courant de " post-post-modernisme » du droit, qui permettrait un renouvellement conceptuel des missions et responsabilités de l'État, lequel dépasserait le rôle de simple régulateur qu'on lui a attribué dans un monde global administré par une main invisible, pour endosser un rôle de gardien des ressources naturelles mais aussi des droits et des intérêts de la population qui dépendent de ces ressources (Morand-Deviller 2012). Autrefois très critiquée, son intervention est de plus en plus considérée comme incontournable et centrale dans la poursuite du bien commun, de l'équité et de l'intérêt général (Arnaud 1998, Arnaud et Farinas Dulce 1998, Halley 2012), notamment en matière de protection de la biodiversité. Les responsabilités associées à cette intervention dans le concept d'État gardien permettent de soulever des questions cruciales comme celle de la capacité des États à prendre en charge ces responsabilités, et souligne ainsi les transformations institutionnelles et normatives à opérer. En tant que tel, il nous semble que le secteur minier ouvre un champ de réflexion intéressant et des questionnements importants pour l'application de ce concept, pour des recherches futures.

\section{RÉFÉRENCES}

Arnaud, A.-J. 1998. Entre Modernité et Mondialisation : Cinq Leçons d'Histoire de la Philosophie du Droit et de l'État. LGDJ, Paris.

Arnaud, A.-J. et Fariñas Dulce, M. J. 1998. Introduction à l'Analyse Sociologique des Systèmes Juridiques. Bruylant, Bruxelles.

Banque Mondiale. 2003. Madagascar-Mining Sector Reform Project. Disponible en ligne <https://preview.tinyurl.com/ya9v76hj>

Baslar, K. 1998. The Concept of Common Heritage of Mankind in International Law. Martinus Nijhoff, La Haye.

Brown-Weiss, E. 1984. The planetary trust: conservation and intergenerational equity. Ecology Law Quarterly 11, 4: 495-582.

Campbell, B. 2010. Ressources Minières en Afrique : Quelle Réglementation pour le Développement? Presses de I'Université du Québec, Québec.

Charte de l'environnement malgache, Loi n 90-033 du 21 décembre 1990 (J.O. n² 2035 du 24 décembre 1990, p. 2540) modifiée par la Loi n 97012 du 6 juin 1997 (J.O. du 09 juin 1997, p. 1171, Édition spéciale et $n^{\circ}$ 2584 du 12 juillet 1999, p. 1479) modifiée par la Loi n 2004-015 du 19 août 2004 et actualisée par la Loi n² 2015-003 du 20 janvier 2015.

Code minier, Loi n 99-022 du 30 Août 1999 modifiée par la Loi 2005-021 du 17 octobre 2005.

Convention des Nations Unies sur le droit de la mer (adoptée le 10 décembre 1982, entrée en vigueur le 16 novembre 1994) 1834 RTNU 3.

Convention pour la protection du patrimoine mondial, culturel et naturel (adoptée le 16 novembre 1972) 1037 RTNU 174.

Convention pour la protection, la gestion et la mise en valeur du milieu marin et des zones côtières de la région de l'Afrique orientale (signée le 21 juin 1985, entrée en vigueur le 30 mai 1996). Disponible en ligne <https://preview.tinyurl.com/y73vjqkp>

Directive du Conseil 79/409/CEE du 2 avril 1979 concernant la conservation des oiseaux sauvages [1979] JO L103/1.

Document cadre de politique minière, Décret n 98-394 du 28 mai 1998 portant définition de la politique sectorielle minière à Madagascar, (J.O. n 2512 du 15 juin 1998, p. 1810).

Fort, F.-X. 2012. L'État fiduciaire et l'obligation de protéger l'environnement. Dans : L'Environnement, notre Patrimoine Commun et son État Gardien : Aspects Juridiques, Nationaux, Transnationaux et Internationaux. P. Halley et J. Sotousek (Eds.), pp 159-180. Yvon Blais, Cowansville, Canada.

Girard, J.F. 2012. La protection des lacs et cours d'eau au Québec : un navire avec plusieurs capitaines. Dans : L'Environnement, notre Patrimoine Commun et son État Gardien : Aspects Juridiques, Nationaux, Transnationaux et Internationaux. P. Halley et J. Sotousek (Eds.), pp 525-558. Yvon Blais, Cowansville, Canada.

Goodman, S. M. \& Benstead, J. P. 2005. Updated estimates of biotic diversity and endemism on Madagascar. Oryx 39, 1: 73-77. <https://doi.org/10.1017/S0030605305000128>

Gouvernement de Madagascar. 2006. Madagascar Action Plan 2007-2012. Disponible en ligne <https://preview.tinyurl.com/y862pcug>

Gouvernement de Madagascar. 2015. Nouvelle aire protégée d'Ampasindava-Galoko-Kalobinono : Plan d'aménagement et de gestion 2015-2020. Ministère de l'environnement, de l'écologie et des forêts, Antananarivo. Disponible en ligne $<$ https://preview.tinyurl.com/ydfgr9qp> 
Gouvernement de Madagascar. 2016. Stratégie et Plans d'Actions Nationaux pour la Biodiversité 2015-2025. Ministère de l'Environnement, de l'Écologie, de la Mer et des Forêts, Antananarivo. Disponible en ligne <https://www.cbd.int/doc/world/mg/mg-nbsap-v2-fr.pdf>.

Halley, P. et Sotousek, J. 2012. L'Environnement, notre Patrimoine Commun et son État Gardien : Aspects Juridiques, Nationaux, Transnationaux et Internationaux. Yvon Blais, Cowansville, Canada.

Halley, P. et Gagnon, C. 2012. Les réformes du droit de l'eau au Québec : un patrimoine commun et son gardien. Dans : L'Environnement, notre Patrimoine Commun et son État Gardien : Aspects Juridiques, Nationaux, Transnationaux et Internationaux. P. Halley et J. Sotousek (Eds.), pp 225-252. Yvon Blais, Cowansville, Canada.

Hufty, M. et Muttenzer, F. 2002. Devoted friends: the implementation of the convention on biological diversity in Madagascar. Dans : Governing Global Biodiversity. The Evolution and Implementation of the Convention on Biological Diversity. P. Le Prestre (Éd.), pp 279-309. Ashgate, Londres.

Jones, G. H. 1992. Droit des trusts. Dans : Droit Anglais. J. A. Jolowicz (Éd.), pp 277-291. Dalloz, Paris.

Les Amis de la Terre France. 2012. Madagascar : Nouvel Eldorado des Compagnies Minières et Pétrolières. Disponible en ligne $<$ https://preview.tinyurl.com/yb8w7b2s>.

Loi affirmant le caractère collectif des ressources en eau et visant à renforcer leur protection, LQ 2009, c. 21 et LRQ, C. C-6.2.

Loi sur les Grands Investissements Miniers, Loi n² 2001-031 du 8 octobre 2002 modifiée par la Loi n²005-022 du 27 juillet 2005.

Moench, M. 2001. Allouer l'héritage commun : le droit à l'eau et son type de gestion en Inde. Dans : L'eau, Patrimoine Commun de I'Humanité. F. Houtart (Éd.). Alternatives Sud 8, 4: 79-104.

Morand-Deviller, J. 2012. L'environnement, notre patrimoine commun. Quelle gouvernance ? Quelles obligations pour l'État gardien? Dans : L'Environnement, notre Patrimoine Commun et son État Gardien : Aspects Juridiques, Nationaux, Transnationaux et Internationaux. P. Halley et J. Sotousek (Eds.), pp 3-16. Yvon Blais, Cowansville, Canada.

Paquerot, S. 2012. Les patrimoines en droit international public : portée et limites. Dans : L'Environnement, notre Patrimoine Commun et son État Gardien : Aspects Juridiques, Nationaux, Transnationaux et Internationaux. P. Halley et J. Sotousek (Eds.), pp 19-34. Yvon Blais, Cowansville, Canada.

Projet Ambatovy. 2006. Environmental and Social Impact Assessment. Vol. B et C. Disponible en ligne <http://www.ambatowy.com/docs/?p=506>

Protocole relatif aux zones protégées ainsi qu'à la faune et à la flore sauvages dans la région de l'Afrique orientale (signé le 21 juin 1985). Disponible en ligne <https://preview.tinyurl.com/y7mpdhhu>.

Rakotondrainibe, M. 2015. Activités minières et protection de l'environnement à Madagascar. Bulletin du World Rainforest Movement 216. Disponible en ligne <https://preview.tinyurl.com/y9kq5pjr>

Randriamiarantsoa, F. 11 mai 2015. Région Anosy : Trois nouvelles aires protégées dans la zone d'ilménite. L'Express de Madagascar. Disponible en ligne <http://fr.allafrica.com/stories/201505112257.html>.

Ribot, C. 2012. Biodiversité et rôle fiduciaire de l'État : les corridors écologiques. Dans : L'Environnement, notre Patrimoine Commun et son État Gardien : Aspects Juridiques, Nationaux, Transnationaux et Internationaux. P. Halley et J. Sotousek (Eds.), pp 369-392. Yvon Blais, Cowansville, Canada.

Sarrasin, B. 2005. La construction des problèmes environnementaux en Afrique subsaharienne : Ia mise en place d'un « diagnostic de Washington sur les ressources naturelles ». Revue Canadienne d'Études du Développement 26, 4: 799-815.

<https://doi.org/10.1080/02255189.2005.9669087>

Sarrasin, B. 2006. Économie politique du développement minier à Madagascar : I'analyse du projet QMM à Tolagnaro (Fort-Dauphin). Vertigo 7, 2: \#2401. <http://vertigo.revues.org/2401>.
Sax, J.L. 1970. The public trust doctrine in natural resource law: effective judicial intervention. Michigan Law Review 68, 3: 471-566. <https://doi.org/10.2307/1287556>

Seagle, C. 2013. "Sauver » la biodiversité en la détruisant. L'exploitation minière de Rio Tinto à Madagascar. LDPI Working Paper 11. <https://Www.iss.nl/sites/corporate/files/LDPI_WP_11_FR.pdf>.

Slade, D. C. 2009. The Public Trust Doctrine in Motion: The Evolution of the Doctrine 1997-2007. PTDIM LLC, Bowie.

UNESCO. 2017. Madagascar. Centre du Patrimoine Mondial. <http://whc.unesco.org/fr/etatsparties/mg>

Valis. 19 avril 2012. Un port dans la Baie de Baly : tortues et aire protégée en cause. Madagascar Tribune.com. <https://preview.tinyurl.com/y82t92b6>

Vincent, P. 2008. Droit de la Mer. Larcier, Bruxelles.

Waeber, P. O., Wilmé, L., Ramamonjisoa, B., Garcia, C., Rakotomalala, D., et al. 2015. Dry forests in Madagascar, neglected and under pressure. International Forestry Review 17, S2: 127-148. <https://doi.org/10.1505/146554815815834822>

Wood, M. C. 2014. Nature's Trust. Environmental Law for a New Ecological Age. Cambridge University Press, Cambridge. 